\title{
Scalable, Shape-specific, Top-down Fabrication Methods for the Synthesis of Engineered Colloidal Particles
}

\author{
Timothy J. Merkel ${ }^{a}, K_{\text {Kevin P. Herlihy }}{ }^{a}$, Janine Nunes ${ }^{a}$, Ryan M. Orgela, Jason P. Rolland ${ }^{b}$, \\ and Joseph M. DeSimone ${ }^{a, b, c, *}$ \\ aDepartment of Chemistry, University of North Carolina at Chapel Hill, Chapel Hill, NC 27599, \\ USA. \\ bLiquidia Technologies, Research Triangle Park, NC 27709, USA. \\ 'Department of Chemical and Biomolecular Engineering, North Carolina State University, \\ Raleigh, NC 27695, USA.
}

\begin{abstract}
The search for a method to fabricate non-spherical colloidal particles from a variety of materials is of growing interest. As the commercialization of nanotechnology continues to expand, the ability to translate particle fabrication methods from a laboratory to an industrial scale is of increasing significance. In this article, we examine several of the most readily scalable top-down methods for the fabrication of such shape specific particles and compare their capabilities with respect to particle composition, size, shape and complexity as well as the scalability of the method. We offer an extensive examination of Particle Replication In Non-wetting Templates (PRINT®) with regards to the versatility and scalability of this technique. We also detail the specific methods used in PRINT particle fabrication, including harvesting, purification and surface modification techniques, with examination of both past and current methods.
\end{abstract}

\section{Introduction}

The design and fabrication of particles with dimensions in the colloidal size scale $(<10 \mu \mathrm{m})$ has become an area of great and continually growing interest for many applications. Particles have been researched and developed for such varied applications as catalysis, microelectronics, photovoltaics, coatings, cosmetics, smart fluids and nanomedicine, among others. These small particles are of fundamental interest not only because of the changes in optical, electrical and other material properties which occur when the material is reduced to the nanometer size scale, but also from other factors such as improved solubility properties or the capability of encapsulating bioactive materials, such as therepeutics or imaging agents, which can be poorly soluble or toxic lacking the protection of the particle. Anisotropic colloidal particles can assemble to form structures which are distinct from the hexagonal close packed arrangements favored by spherical particles and are of interest in the fields of memory storage, optical electronics, photonics and sensors. 1

Considerable effort has been devoted to the development of fabrication methods which can mass produce colloidal particles with fine control of size, shape, composition, cargo and surface chemistry. We submit Particle Replication In Non-wetting Templates (PRINT) as a readily scalable method for the fabrication of such monodisperse colloids with precise control over size and shape and with broad capabilities with regards to material composition

\footnotetext{
”To whom correspondence should be addressed: desimone@unc.edu.
} 
and chemical anisotropy. In addition to PRINT, we will present several other top-down particle fabrication methods which have excellent potential for scalable production of monodisperse, shape-specific particles on the colloidal size scale.

Two broad approaches are amenable for the fabrication of anisotropic particles; bottom-up and top-down techniques. Bottom-up approaches begin at the atomic or molecular scale and build up to the desired particle size, while top-down methods process a material on the desired size scale. The most commonly employed methods for the production of mass quantities of particles on the colloidal length scale are bottom-up synthetic approaches such as emulsion polymerization. In a typical process, a monomer is emulsified via rapid stirring in a mobile phase which contains an initiator and a surfactant. Upon heating to activate the initiator, spherical particles are nucleated in the surfactant micelles and grow to the desired size. Particles obtained by emulsion methods are typically spherical and can vary in size from tens of nanometers to as large as several microns in diameter. The particle size and the molecular weight of the polymers formed in emulsions are controlled with parameters such as the surfactant concentration and reaction temperature. Surfactant adsorbed onto the surface of these particles can be difficult to remove if undesired. While this method is extremely scalable, the particles fabricated in this way are typically spherical in shape and are fairly polydisperse.

There is a growing need to generate particles with a diversity of non-spherical shapes. Complex particle shapes are desirable for a range of applications, including self-assembly2, photonic materials3, and microelectromechanical systems (MEMS).4 Particles with nonspherical shape are of increasing interest for biomedical applications such as drug delivery, where the rod-like or corkscrew morphologies possessed by many viruses and bacteria are suspected to have derived an evolutionary advantage from their specific shapes.5 For many applications, the ability to control particle size and shape is of vital importance, and the ability to manufacture large quantities of such size and shape-specific particles is a crucial factor in the utility of the fabrication method. For a particle fabrication method to prove industrially useful, it must be scalable and capable of producing usefully large quantities of particles. For example, a particle tested for efficacy as a drug carrier will have very different production needs in the laboratory setting compared to the industrial scale. The fabrication of milligram quantities of particles suffices for testing in the lab, while commercialization of this technology would require production of gram and kilogram quantities to satisfy the needs of phase 1 or 2 clinical trials.

While the focus of this paper is on top-down methods, a brief review of bottom-up particle fabrication methods may be instructive by way of comparison. Bottom-up approaches are readily scalable, but can lack fine control of particle size and dispersity, and can be limited in the variety of shapes which can be produced. Crystals of many metals and metal-oxides can be grown into both spherical and anisotropic shapes such as cubes, rods, discs and faceted polyhedra using nucleation and arrested growth strategies. 1 More complex branched structures can be formed by sequential growth of dots and rods of different materials. 6 Selective crystallization and deposition methods have been developed to produce prisms, rods, arrows and teardrop shaped crystals of gold, silver and cadmium selenide, respectively. $7^{-} 9$ Such nucleation and growth methods are limited to inorganics and the shape selectivity is highly dependent on the material and its crystal structure. Further, these inorganic materials often lack the capability to encapsulate a cargo or undergo surface modification.

Bottom-up approaches for the fabrication of organic particles have received a great deal of attention for use in biomedical applications such as imaging, gene or drug delivery. These methods chiefly rely on self-assembly to create hollow particles such as micelles, vesicles, liposomes, and polymersomes which are able to encapsulate a cargo in their otherwise 
hollow interior. In a typical preparation, molecules are synthesized which possess both hydrophilic and hydrophobic domains. Upon exposure to water, the molecules orient themselves to present their hydrophilic portion to the water, while the hydrophobic domains orient inward, resulting in a sphere with a hydrophobic pocket. The hydrophobic interior is able to encapsulate a hydrophobic cargo. While spherical particles have been the standard, increasing attention has been directed towards higher aspect ratio particles which are achievable with control of the relative length of hydrophobic and hydrophilic domains.5, 10 Recently, Discher, et. al. explored filamentous polymersomes called filomicelles for their circulation persistence and ability to encapsulate and deliver chemotherepeutics. The filomicelles were prepared from block copolymers with lipid-like amphiphilicity, but a more symmetric ratio of hydrophilic to hydrophobic blocks than is found in lipids. Filomicelles were prepared with a hydrophilic block of poly(ethylene oxide) and hydrophobic block of either poly(ethylethylene) or poly(caprolactone). These filamentous particles persisted in the circulation about ten times longer than spheres with similar surface chemistry, and were successful in encapsulating and delivering a hydrophobic chemotherapeutic to tumored mice.10 One criticism of these self-assembled systems is the dynamic nature of the particles so assembled. With membrane components held together with attractive forces rather than with covalent bonds, the structures generated can add or lose components, making their size and shape more fluid than may be desired. The dynamic nature of these self-assembled systems has been addressed by Wooley et al. with shell-cross linked knedel-like particles (SCKs).11 These block copolymer micelles are modified with reactive groups on the surface which can be chemically cross-linked after self-assembly, giving superior stability to their structure.

\section{Top Down, Scalable Methods for Fabricating Shape-specific Particles}

Bottom-up fabrication methods tend to be readily scalable to large batches, but typically offer limited control over size, size distribution and particle shape. While "crude" top-down methods, such as milling or grinding of bulk materials, have found utility in industry, they typically offer very little control over these same parameters of particle size, size distribution and shapes deviating greatly from the spherical. Several top-down particle fabrication methods are excellent targets for mass production of shape-specific particles, having the potential for superior control over the above parameters, with the potential for scalability. In addition to the PRINT method favored by the authors, hard-template methods, microfluidic reactors, and particle stretching all show promise in this regard (Table 1).

\section{Hard-template synthesis of nanoparticles}

For the last twenty years, hard-template methods have been used extensively to prepare nanowires, nanotubes and nanorods. 28 In this technology, porous templates are filled with one or more materials to fabricate monodisperse nanoparticles. The two most common templates used are anodized aluminum oxide (AAO) and track-etched polymer membranes. $\mathrm{AAO}$, formed from the electrochemical oxidation of aluminum in an acid electrolyte, has a high density of uniform, parallel pores (as high as $10^{11}$ pores $/ \mathrm{cm}^{2}$ ) regularly spaced on a hexagonal lattice. Templates have been fabricated with pore diameters as small as $5 \mathrm{~nm} .29$ Track-etched polymer membranes are formed via the bombardment of polymer films, such as polycarbonate, with nuclear fission fragments to create damage tracks which are then chemically etched into pores. These polymer templates contain randomly distributed, uniform-diameter pores, with pore densities approaching $10^{9}$ pores $/ \mathrm{cm}^{2}$, and pore diameters as low as $10 \mathrm{~nm}$. In this case however, the pores are not parallel, and so may intersect. Traditionally, the templates are filled via electrochemical means. This is accomplished by first depositing a sacrificial metal layer, such as gold or silver, on one side of the template for electrical contact. Then, the material or materials of interest are electrochemically deposited into the pores of the template, for example metals can be synthesized using the 
appropriate electroplating solution, and conducting polymers can be synthesized via oxidative polymerizations. After deposition, the template can be dissolved, for example the AAO dissolves readily in acidic or basic solutions. This yields an ensemble of nanoparticles connected at the base to the substrate metal. It is occasionally useful to leave the nanoparticles connected for electronic applications however, the connecting sacrificial metal layer can also be dissolved to generate a monodisperse suspension of high aspect ratio nanoparticles. Both tubes and solid wires or rods can be prepared depending on the surface treatment of the template. For example, gold nanowires can be synthesized galvanostatically in AAO, however, if the template is first treated with (2-cyanoethyl)triethoxysilane, the gold preferentially deposits on the walls of the template pores and thus forms nanotubes, instead of solid nanowires.30 Additionally, while the nanotubes are generally prepared with uncapped ends, they can be synthesized with one end capped.31 This has interesting implications on the ability of nanotubes to act as effective biological delivery vectors. 31 Non-electrochemical methods have also been developed to fill the templates, such as solgel32 and layer-by-layer33 approaches for the fabrication of semiconductor (cadmium selenide, zinc oxide, graphitic) nanoparticles, and nonconducting polymer particles.34

Remarkably, Martin and coworkers13, 14 have extended the template method to the fabrication of biological nanoparticles such as DNA and protein nanotubes. For the case of DNA nanotubes, this was made possible by pretreating the template pores with an alternating $\alpha, \omega$-diorganophosphate $(\alpha, \omega$-DOP) $\mathrm{Zr}(\mathrm{IV})$ chemistry. The first DNA segment binds to the $\mathrm{Zr}(\mathrm{IV})$ through the phosphonate end of the DNA strand. Subsequent DNA segments are incorporated into the nanotube through hybridization with the preceding DNA segment. Thus, the resulting DNA nanotube has an outer skin comprised of the ( $\alpha, \omega$-DOP) $\mathrm{Zr}(\mathrm{IV})$ layer.13 A similar alternating chemistry approach was used in the fabrication of protein nanotubes - the AAO pores were first treated with 3-amino propylphosphonic acid, followed by the protein immobilization agent, glutaraldehyde. Protein then reacted with the glutaraldehyde, and additional alternating layers of glutaraldehyde and protein were deposited to thicken the nanotube walls.14 One crucial factor in these biological systems is the release of the nanotubes from the template since the AAO template is dissolved under acidic or basic conditions. For the release of these nanotubes, the template was dissolved under very mild acidic conditions ( $1.5-5 \%$ phosphoric acid) 13,14 at $0{ }^{\circ} \mathrm{C}$ for 24 hours. The biological activity of the nanotubes was confirmed after release. Though in the case of the protein nanotubes, it was noted that many of the tubes were broken during the dissolution and filtration steps. 14

One major advantage of the template method is that segmented or multicomponent nanoparticles can be straightforwardly fabricated (Figure 1).12, 35 This has led to an impressive breadth of research into the area of post-fabrication modifications of segmented nanoparticles. Multicomponent nanorods can be selectively functionalized for different multiplexing, sensing and self-assembly applications.36 37, 38 Mirkin, et al. developed onwire lithography (OWL) to create nanowires with nanoscale gaps along the length of the wire. For example, a segmented nanowire with alternating gold and nickel segments was synthesized electrochemically in an AAO template.37 After the nanowires were released and dispersed onto a substrate, gold or silicon dioxide was coated onto one side. The rods were then redispersed and the nickel was etched, resulting in nanowires with predefined gaps and disks. These architecturally complex structures have been found to have very interesting properties useful for nanoelectronics 12 and surface-enhanced raman scattering (SERS). 38

It is evident that the hard-template method is useful for the synthesis of monodisperse nanoparticles. In fact, it is currently the preferred method for synthesizing very high aspect ratio nanoobjects. $12 \mathrm{~A}$ wide range of chemistries can be applied, and it is straightforward to 
fabricate particles with multiple chemistries spatially resolved within the particles. One concern with this method is that as the particles are released upon dissolution of the template and metal substrate (in the case of electrodeposition), the particle chemical composition must be compatible with the solvents used, usually acids and bases. Another drawback of this process is the limitations in nanoparticle shape - most nanoparticles have been synthesized with cylindrical geometries. Furthermore, the issue of scalability is somewhat questionable as virtually all research has been conducted on relatively small scales (approximately 1 square inch), so while theoretically this approach can be high-throughput, this has yet to be truly developed. Among other factors, scale-up will be dependent on the availability of large area, high density templates.

\section{Microfluidic particle fabrication}

One emerging strategy for the fabrication of particles with control over particle size and shape is fabrication with microfluidic devices. In a microfluidic reactor, minute amounts of liquid flow through micron sized channels which can be designed to produce monodisperse emulsion droplets. These emulsion droplets are then solidified to form particles. Most methods for droplet formation in microfluidic devices are passive ones which rely on the liquid flow field deformations for droplet formation rather than mechanical actuation or moving parts.41 Passive droplet formation stems from several different device architectures such as multiphase flows at T-junctions42, flow focusing channels43, or with coflow based systems 44 which exploit the laminar nature of fluid flow at the low Reynolds numbers associated with the small size of the channels. Because of the nature of these fluid dynamics, liquid-liquid interfaces for parallel flowing liquids experience only diffusion based mixing and sharp boundaries are maintained, allowing for the formation of droplets with small size distributions.

The material of the droplets determines the method by which they can be solidified to form particles. Droplets can simply be cooled from a liquid to a room temperature solid phase, or solvent evaporated from a dissolved polymer. Photo or thermal polymerization of droplets with reactive groups is another method for solidifying droplets. Solidification of droplets by means of a chemically driven polymerization reaction, such as the condensation of amines and carbonyl chlorides, has also been reported.45 Essentially, the droplets must be composed of a material which is capable of photochemical, thermal or chemical curing or they must be readily melt or solvent processable materials.

Shapes produced in these channels have been limited to spheres, cylinders and spherically derived shapes such as discs and ellipsoids. More complex and chemically anisotropic shapes such as hemispheres 46 , core-shell 46 and janus particles 18 were produced by confining the droplets with the geometry of the device and with coflowing laminar streams, though these remain derivatives of the original spherical shape. Microfluidic techniques have readily produced particles on the size range of $10-100 \mu \mathrm{m}$, though reduction much below this size scale has remained a limitation for the technology. 15

Recently, the Doyle group has developed photolithographically coupled approaches, which have produced more complex shapes by use of a photolithographic mask.20, 47 In continuous flow lithography (CFL), a flowing stream of monomer in a polydimethylsiloxane (PDMS) channel was exposed in mask-defined shapes with short bursts of UV light. Particles were crosslinked by the UV light exposure almost instantaneously, and did not plug up the channel due to the oxygen mediated inhibition of polymerization at PDMS surfaces, which left a thin layer of unpolymerized monomer at the channel walls.48 The output of particles was continuous and the shape was defined by the limits of the lithographic mask (figure 2). Exposures across several fluid streams resulted in the formation of janus type particles with two or more chemically distinct regions on each 
particle.20 CFL systems have some limits to resolution and speed however, as higher flow speeds and smaller particle sizes caused blurring or smearing of the particles formed in the moving stream of polymerizable fluid. Higher resolution has been achieved with the use of stop-flow lithography (SFL), a process during which the flow of monomer was stopped briefly during each exposure. This process enhanced the resolution of the lithographic technique compared to CFL, but was limited somewhat by the time taken by the flowing liquid to stop and restart. Complex shapes have been formed with high resolution in the micron scale, including a range of concave and convex sided pyramids, rectangles, and spheres, and rectangular toroids - all with multiple, chemically distinct phases possible. 47

Microfluidic methods have demonstrated their versatility in terms of particle shape and chemical anisotropy of the particles produced. The emergence of CFL and SFL techniques have increased the resolution of this technique though there are material limitations; particles made in this way have been limited to polymerizable fluids and solvent choice has been limited due to the swelling of the device material, PDMS, in many solvents. Another limitation has been the size of particles which can be produced with these methods. Particles on the order of 3-100 $\mu \mathrm{m}$ were readily fabricated, but sub-micron particle sizes remain a challenge for microfluidic technology. The scalability of this technique is somewhat in question, though the autonomous nature of the technology makes it a tempting target. In a simple CFL device, a throughput of near 100 particles per second was reported.20 Simply stacking many devices together to scale-up production of particles, while the simplest strategy, has been seen as impractical due to the complications in linking up all of the required tubes and pumps required for multiple devices. 49 With typical flow rates in the range of $1000 \mu \mathrm{L} /$ hour for droplet based reactors, an industrially useful output of 1 liter of material per hour would require 1000 reactors chained together, which seems feasible. More complicated devices, which have as many as 128 droplet producing cross-junctions, have been fabricated to facilitate scale up and reduce the number of devices needed for mass production of particles.16, 50

\section{Particle Stretching}

Spherical particles prepared by bottom-up methods such as microemulsion can be manipulated to produce anisotropic shapes. This particle stretching method initially was used to deform polystyrene (PS) particles which were embedded in a polyvinyl alcohol (PVA) film. Upon heating to a temperature at which the particles could deform, the film was stretched, then rapidly quenched, yielding ellipsoidal particles upon dissolution of the PVA fim.51

Recently, Champion et. al. reported the formation of complex shapes via a similar method (figure 3). In this method, as before, (PS) particles were embedded in a PVA film. The PS particles were liquidized by addition of heat or solvent, allowing the PS to flow, filling void spaces in the surrounding film, or to stretch with the film due to hydrogen bonding based attraction to the surrounding film. Manipulation of the PS particles required exposure to temperatures on the order of $140{ }^{\circ} \mathrm{C}$ or organic solvents (toluene), which places some limits on the encapsulation of cargos by these particles. Careful modifications to the stretching protocol allowed for selective formation of over twenty distinct particle shapes upon solidification of the particles by cooling or removal of solvent and subsequent release from the PVA film.52

This particle stretching method has also been demonstrated on particles composed of PLGA, a hydrophobic polymer which has utility in drug delivery applications. PLGA particles were deformed from their initial spherical shape at much lower temperatures than was possible with PS particles, due to the lower glass transition temperature of this material $\left(40-60{ }^{\circ} \mathrm{C}\right.$ for PLGA versus $90^{\circ} \mathrm{C}$ for PS). Particles processed in this way were able to encapsulate a 
fluorescently labeled protein (66kDa FITC-labeled bovine serum albumin) with no leaching of the protein during stretching of the particles, suggesting that the processing methods were mild enough to accommodate many such biological cargos.5

One distinct advantage of the particle stretching technique has been the ability to compare differently shaped particles with identical volumes by preparing different particle shaped from the same stock of spherical particles. Material compatibility is a concern for this method, as the bulk of the literature deals with PS and PLGA based particles - though one can imagine that many materials could be manipulated in this way by adjusting the conditions used to fluidize the particles and the material in which the particles are embedded. This method is versatile with respect to particle size, as spherical PS particles with diameters from $60 \mathrm{~nm}$ to $100 \mu \mathrm{m}$ have been subjected to these stretching protocols. Scalability of the method remains to be seen, as particles have only been produced on a lab scale. Particle yields of $10^{8}-10^{10}$ particles per stretching apparatus have been reported5, though thicker or stacked films and larger stretching mechanisms could increase throughput. Polydispersity of the particles prepared by stretching methods has reflected the polydispersity in the spherical particles before stretching. Careful selection of a spherical particle source could lead to the formation of more monodisperse populations of stretched particles.

\section{Photolithographic Methods}

Photolithography, the workhorse of the semiconductor industry for high volume manufacturing53, has also been directly applied to the fabrication of discrete monodisperse colloidal particles.23, 54, 55 There are four major steps to the process. The first is the spin coating of a sacrificial layer, followed by a photoresist layer on a silicon wafer. The particle will be composed of the photoresist material, and so the thickness of that layer will determine the particle height. Second, the photoresist is exposed to ultraviolet light through a mask with the specific pattern of interest. This exposure step can be done in an automated UV lithographic projection exposure system, or stepper.23 In the case of a negative photoresist, the exposed areas of the photoresist are rendered insoluble after post-exposure baking steps due to cross-linking. The third step is development of the photoresist, where an organic solvent dissolves the unexposed regions leaving discrete photoresist particles on the sacrificial layer. The final step is the dissolution of the sacrificial layer to release the particles. A simple illustration of this process is shown in Figure 4A.

Both Mason et al.23 and Stroock et al24. have reported using this type of approach to generate highly monodisperse, shape-specific colloidal particles. Stroock et al.56 demonstrated the ability to tailor the surface roughness of the particles by modifying the development conditions (Figure 4B). Mason et al.23 illustrated the shape versatility of this process by generating particles with a wide range of shapes, including all 26 letters of the English alphabet. The fabrication of the lithographically-derived particles, or LithoParticles as coined by Mason, was also found to be amenable to the incorporation of both organic dyes and nanoparticles such as iron oxide. It was even feasible to fabricate hybrid bilayer Janus particles, through the successive exposure of two different masks. Even though this photolithographic process has been optimized for photoresists, it is possible to use other UV-reactive systems. For example, microparticles composed of methacrylated hyperbranched polyglycerols were fabricated directly using photolithography. 57

There are many clear advantages to this type of processing: there is excellent shape fidelity down to the submicrometer level, and the process can be high throughput. For example, if a stepper with an automated wafer handling system is used, it is possible to expose one fiveinch wafer every minute to yield about $10^{8}$ particles/ minute23 (this estimate does not, 
however, consider the post-exposure bake, development and release steps). It was envisioned that by incorporating all the equipment necessary for making the particles into a robotically automated track system, a continuous rate of $10^{8}$ particles per minute is achievable.23 This however creates a major stumbling block for using this process as a large scale particle fabrication technology because the enormous capital required to continuously run such an expensive lithographic exposure system is extremely daunting. Another major issue with this type of processing is the limitations in the chemistries that can be used in the particle fabrication. These issues have led to the development of alternative routes to LithoParticle production that take advantage of templating, where only the initial reusable template requires photolithography. 58

Other direct photolithographic approaches for particle fabrication include the use of interference lithography, where the constructive and destructive interference of multiple laser beams at the surface of photoresist generates patterns without the use of a mask.59 This is a benefit as high resolution masks can be fairly expensive to fabricate. Usually, the projected patterns are limited to regularly spaced lines or dots59 - this strategy has been used to fabricate cylindrical particles with varying aspect ratio from 0.4 to 10.55 However, Thomas and coworkers54 have shown that this process can be used to fabricate multivalent 3D colloidal particles (Figure 4C). These complex shapes are extremely difficult to fabricate by other top-down fabrication processes.

\section{Imprint Lithography Methods}

Imprint lithography based methods have emerged in recent years as promising techniques for scalable fabrication of shape specific colloidal particles. Soft lithography based techniques, which were pioneered by Whitesides et al. in the late 1990's, begin with the use of a rigid template which is used to fabricate an elastomeric mold, which can then be used to replicate the shape of the original template in a molding or stamping process.61 Imprint methods were first envisioned as a high-resolution, low-cost alternative to traditional photolithograpic techniques for micro- and nanoscale pattern replication rather than fabrication of distinct particles. Particle fabrication has been a challenge due in large part to the persistent formation of a residual layer of molded material, called a flash layer, which connects the potential particles.62 Much attention has been directed towards the removal or avoidance of this flash layer with the goal of particle fabrication. Step and flash imprint lithography (S-FIL) uses a rigid, UV transparent template as a mold material, creating an embossed film of particles from a UV curable material. The interconnecting flash layer is removed by an etching step, with dissolution of a sacrificial layer yielding distinct particles. 63 Removal of the flash layer via an oxygen plasma etch may be undesirable for some particle compositions or cargos, though etch times from 20-90 seconds have been reported for effective particle release, and the biological activity of an encapsulated protein was maintained after 20 seconds of etching. 63

Utilization of the traditional mold material for soft lithography applications, a crosslinked poly (dimethyl siloxane) (PDMS) elastomer, typically results in particles connected by such a flash layer. Recently, microparticles have been fabricated with PDMS molds without formation of a flash layer by careful applications of photocurable acryalte resins as well as aqueous and organic solutions of polymers, to the molds.64 Surface treatments can increase or decrease the hydrophilicity of the mold, facilitating mold-filling, mold de-wetting, and particle release.65 PRINT technology (figure 5), which utilizes a fluorinated elastomeric mold in an imprint lithographic technique, allows for facile fabrication of distinct particles without formation of a flash layer while maintaining many of the advantages of the soft lithography technique while avoiding some of the difficulties associated with PDMS.66 


\section{Particle Replication In Non-wetting Templates (PRINT)}

While a number of published manuscripts are available describing a wide variety of particles fabricated using the PRINT technique (figure 6) $27,66,68^{-} 72$, the details and rationale behind particle fabrication can be confusing. With this in mind, the goal of this section is to revisit past particle fabrication methods, to examine the current state of the art of the PRINT technique, to provide a clear picture how the technique works, and to look at why the PRINT technique can be readily scaled up.

In 2004, Rolland et al. reported the synthesis of a new perfluoropolyether (PFPE) based elastomer73, a material that proved to be more effective than PDMS as a template for soft lithography based particle fabrication. Photocurable PFPE (Fluorocur ${ }^{\mathrm{TM}}$, Liquidia Technoligies Inc., NC) was synthesized by endcapping a fluorinated PFPE diol (Solvay) with isocyanatoethyl methacrylate to form PFPE dimethacrylate. The end-functionalized fluorinated oil had a positive spreading coefficient allowing it to completely wet and envelop the delicate details of a master template. Curing of the oil was accomplished under UV light $(365 \mathrm{~nm}, \sim 10-15,000 \mathrm{~mJ} / \mathrm{cm} 2)$ after the addition of a photoinitiator. The resulting PFPE elastomers have three distinct advantages over silicone templates for use in soft lithography. First the low surface energy of the elastomer $(8-10 \mathrm{dyn} / \mathrm{cm}) 74$ facilitates the removal of an excess flash layer. Other lithographic methods typically require an etch step to remove this layer, complicating the scale up of these methods. 75 Second, PFPE was observed to be compatible with a number of organic solvents that were incompatible with silicone molds.73 It was found that, while both Sylgard 184 (a silicone material) and PFPE molds exhibited negligible swelling in the presence of water and methanol, Sylgard 184 molds were permeable to toluene and dichloromethane. An examination of several other common solvents and polymerizable organics showed a similar trend, with PDMS coupons generally showing a higher retention of these organic fluids (table 2). With the elimination of swelling, master-to-replica fidelity was improved and the reproduction of much smaller feature sizes was possible. In 2006, Maynor et al. showed that a variety of nanoscale artifacts could be reproduced using the PRINT technique including $60 \mathrm{~nm}$ adenoviruses, 45 $\mathrm{nm}$ polymeric toroidal micelles, and single walled carbon nanotubes with heights as low as 2 $\mathrm{nm} .74$ Third, the highly fluorinated nature of the mold (similar to that of Teflon ${ }^{\mathrm{TM}}$ ) facilitated the removal of particles from the mold. In contrast, other soft lithography methods required surface modification of the molds to facilitate particle removal.65

Initially, mold fabrication was performed by drop casting $20-30 \mathrm{~mL}$ of Fluorocur resin onto a 6-8 inch diameter patterned silicon master template that was then cured photochemically in a UV oven.66 Molds were released from the template by slowly peeling them back from silicon wafer using PTFE tweezers so as not to scratch the fragile surface features. Because of the delicate nature and the high cost associated with using large quantities of Fluorocur Resin for the thick molds, the scale of particle production was limited. With this in mind, a proprietary roll-to-roll system capable of producing up to one thousand square feet per day of PFPE molds on a flexible poly (ethyleneterephthalate) (PET) backing, was recently engineered by Liquidia Technologies. 25 The new process yields mechanically robust molds and uses a reduced amount of Fluorocur Resin, making the process less costly and highly scalable.

\section{Mold Filling}

Filling of any patterned mold is a process that relies heavily on surface energies of the materials in play. In order to effectively fill the cavities, a pre-particle solution should have the ability to partially wet the surface of the mold. Solutions capable of wetting the surface of a mold are drawn into the cavities of the mold by capillary forces. During the fabricating of embossed films removal of excess solution is not a concern, but in particle fabrication, in 
which individual structures rather than one monolith are desired, management of excess preparticle solution is crucial. After creating a solid embossed film, the step and flash imprint lithography (S-FIL) method, recently commercialized by Molecular Imprints, Inc., removes the flash layer using a "breakthrough" etch to produce individual surface features. For high throughput particle fabrication, however, it is desirable to eliminate any etching steps as they are time intensive and not amenable to a continuous process. Using the PRINT method, fabrication of discrete particles was made possible by the removal of excess solution prior to the solidification of particles in the mold. Excess pre-particle solution was removed by first laminating the filled mold with a high surface energy material (i.e. PET, surface energy = $42.1 \mathrm{mN} / \mathrm{m}$ ) 77 and second, peeling back the laminate material. Capillary forces trap the liquid in the mold while excess solution adheres preferentially to the high surface energy material and is wicked away leaving isolated reservoirs of pre-particle solution, thus eliminating the need for a scum removal step.

Like surface energy, other factors such as film uniformity and film thickness of the preparticle solution play important roles in effective large scale particle fabrication. Uniform films were drawn on a high surface energy sheet using a metal Mayer rod with grooved surface features and a mechanical film coating instrument (R.D. Speicialties Inc). Film deposition by Mayer rod is highly amenable to a continuous roll-to-roll process making it possible to easily lay thousands of feet of uniform film on a substrate. Film thickness was dictated by both the grooved feature size of the Mayer rod and the concentration of the preparticle solution.

Unlike patterning on a solid substrate, or with a rigid patterned template which can be time consuming, patterning of the pre-particle solution can be done in a continuous fashion when the template and pre-particle solution are both on flexible backing. Other particle fabrication methods like S-FIL involve a step by step procedure in which a master template is brought into contact with a solid substrate in the presence of a pre-particle solution, a step that can damage the master template if performed without care. In the PRINT process, after casting a uniform film on PET as described above, the PET was laminated to an empty mold using an in-house single-nip lamination system. Lamination conditions (pressure, speed, temperature, and humidity) were optimized for each new class of particle theoretically allowing the continuous fabrication of hundreds of feet of laminated mold. Monomer-based pre-particle films that flowed freely into the cavities of the mold and had low vapor pressure were laminated at room temperature.71 Volatile monomers and other small molecule systems required that the lamination temperature be reduced and that the lamination take place in glove bag or other controlled environment. Polymeric films like poly(ethylene glycol) (PEG) or poly(lactic-co-glycolic acid) (PLGA) sometimes required heat to raise the temperature of the film above the glass transition temperature of the polymer allowing the polymer to flow into the mold.

\section{Curing/Solidification}

While the majority of "Top Down" particle fabrication processes rely on photochemical curing, particle solidification using the PRINT can be quickly accomplished by a variety of different methods. Monomer systems incorporating a photochemical initiator such as 1hydroxycylohexyl phenyl ketone or 2,2-diethoxyacetophenone (HCPK and DEAP, Aldrich) were cured in a UV chamber.72 Some monomeric pre-particle systems that were sensitive to light were instead cured thermally using a temperature sensitive initiator such as 2,2-bisazobutronitrile (AIBN, Aldrich). Polymeric systems cast from a solvent were dried under vacuum while neat polymeric systems were dropped below their glass transition temperatures. Small molecule and protein based "biological" systems that were cast from a solution were typically lyophilized.27 
With the PRINT process, particle solidification, regardless of the chemical process, was typically performed one of two ways: either closed-faced, with a PET sheet laminated to the surface of the filled mold or open-faced70 with the filled mold exposed to a $\mathrm{N}_{2}$ purge. Solidified particles trapped in the mold or bound to a harvesting layer of PET could be harvested immediately or wound up and stored until harvesting at a later date.

\section{Harvesting}

With the PRINT method, removing particles from the mold was initially performed by physical agitation of the particles with the sharp end of a glass slide.69 A number of problems were associated with this harvesting technique. First, this method was found to damage the surface of the molds creating large chunks of PFPE mold that would have to be filtered out later. Second, scraping dry particles could cause them to aerosolize. Third, the harvesting process was not scalable. New harvesting techniques have been developed that are more gentle, safe, and scalable. If solidified with an open face as noted above, the particles in the mold could be laminated to an adhesive layer such as polyvinyl pyrrolidone (PVP) or cyanoacrylate on a flexible or rigid backing such as PET or glass slides. Once the adhesive has solidified, the mold is peeled away from the substrate revealing an array of free-standing particles. The adhesive can then be dissolved and the particles collected in solution. If the particles are solidified with a closed face (laminated to PET), the particles will adhere to the PET once the mold is peeled back. The free-standing particles can then be harvested by lamination to another adhesive layer or by physical agitation with a soft rubber cell scrapper in the presence of a solvent. As with film fabrication, mold filling, and particle solidification, particle harvesting, as a continuous process, is possible using the PRINT method.

\section{Purification}

Regardless of the fabrication method, after harvesting, particles made from "Top-down" techniques generally require some form of purification to remove debris or residual compounds from the fabrication process, and to prepare particles for their intended use. As mentioned above, when using AAO as a template, particle fragmentation often occurred.14 Even particles that only required minimal manipulation by physical stretching on a sheet of PVA required multiple rinsing steps to remove excess PVA from solution.52 Fortunately, a wide variety of purification methods are currently available and it is usually possible to find at least one method that fits well with a particle fabrication technique. While all purification methods have their own distinct advantages and disadvantages, some are intrinsically slow (based on batch to batch processing) while others are much faster (based on automated continuous processes).

Dialysis (>100,000 MWCO, Float-a-lyzer, Spectrum Labs, Inc) was originally used for PRINT particle fabrication to remove impurities such as sol fraction and to exchange buffer solutions. This method was found to be a relatively slow process and particles were often physisorbed to the membrane surface. In a typical purification, spent solvent had to be switched for clean solvent at least three times over the course of 1-2 days.

Centrifugation is perhaps the simplest method of particle purification and it is used widely as a quick method to rinse particles and separate various sized populations of particles gravimetrically.68 In centrifugation, particles are spun out of solution under high centrifugal forces. As particle size decreases, the centrifugal speed and the time required to effectively pellet out particles effectively increases. Particle yield can be reduced when centrifugation is halted prematurely. As such, purification of small stable particles, less than a few hundred nanometers in diameter, can be quite time consuming requiring hours or days to fully pellet a sample. Other problems including irreversible aggregation may be encountered when 
particles are centrifuged for long periods ( $>1 \mathrm{hr})$ at high speed $(14,000 \mathrm{rpm} / 20,000 \mathrm{G})$. Additionally, resuspending pelleted particles by vigorous mixing and/or sonication can damage particles composed of less robust matrix materials or with high aspect ratios as well as fragile cargos, such as proteins and other biologically active cargo, and should be performed with care.

Centrifugal filtration using $0.1 \mu \mathrm{m}$ cutoff poly(vinylidene fluoride) (PVDF) membranes (Microcon-MC, Millipore) proved to be an efficient alternative to standard centrifugation. Lower speeds are required than normal filtration and therefore aggregation is typically reversible. Unfortunately, once spun down, particles are partially adhered to the PVDF membrane. The strength of particle adhesion to the membrane depends strongly on both the chemistry of the particles as well as that of the membrane. Removal of particles from the membrane was found to be optimal when the membrane was physically removed from its housing and sonicated in solution. While a variety of polymeric membranes and pore sizes are available making this technique adaptable for a variety of particle fabrication methods, particle loss is often dramatic (as much as 50\% loss of mass), and the batch process with repeated rinsing can be slow and therefore it is not ideal for high throughput particle fabrication.

Filtration using indirect or tangential flow filtration (TFF) systems appeared to be the most promising approach for particle purification. In TFF filtration systems such as KrosFlo (Spectrum Labs, Inc) particle solutions traveled tangential to the membrane. Both diafiltration as well as concentration are possible without changing particles from one set up to another. Particle loss by adsorption to the membrane and to surfaces was reduced as particles were not forced to interact directly with the membrane or multiple vials and/or pipet tips. Stirred Cell filtration (Millipore), another indirect filtration method, was also examined. While this method of purification appears promising, inadequate stirring often leads to filters clogged with particles creating prolonged filtration times. Additionally, if the cell is allowed to run dry dramatic loss of particle yield is observed due to irreversible adhesion of the particles to the filter membrane.

Other methods of particle purification are also under development. Magnetic purification for example is currently being used for particles containing magnetic cargo. While it was strictly limited to magnetic particles, this method, using magnetic columns (Minisep, Miltenyi Biotec) appeared promising for purification and other functionalized particle applications. In addition to magnetic filtration, Flow Field Fractionation (FFF) is of interest for future purification and separation of particles as particle aggregation is not as prevalent as in other centrifugal techniques.

\section{Surface Modification}

One unique feature of template based particle fabrication is that solidified particles, either in the mold or on a harvesting layer of PET, are easily subjected to a number of regiospecific chemical modifications.67 Chemical modification of particles while in the mold made it possible to manipulate one face of the particle. Once removed from the mold, modification of particles on a harvesting layer allowed the manipulation of the remaining sides of the particle. This is an exciting development for a number of fields of research including nanoand microrobotics 78,79 and targeted drug delivery.67 Ongoing research in our lab is focused towards directed cellular uptake of regio-modified particles as well as the beginnings of shape-specific building blocks for directed assembly in smart fluids and micro-robitics applications. 


\section{Summary}

There is a growing, and widening, demand for monodisperse, shape-specific colloidal particles for both the academic and industrial sectors. As yet, no standard particle fabrication method has arisen to fulfill this unmet need. We have identified and examined several candidate top-down particle fabrication methods, each of which is capable of producing shape specific particles composed of a range of materials with the highest potential for scalability. In particular, we have scrutinized some of the materials and methods used in PRINT particle fabrication, modification and purification, many of which may be of general use for particles derived by other methods.

Each of the particle fabrication methods discussed herein possess inherent strengths and weaknesses to their versatility and capabilities. Hard template methods seem ideally suited for the formation of high aspect ratio particles though other shapes have not been demonstrated. Particle stretching methods have the advantage of modifying prefabricated spherical particles, which can be more readily mass-produced, to achieve non-spherical shapes. Microfluidic methods have, perhaps, been most broadly developed of those mentioned here and have been demonstrated to have excellent scalability and versatility. Photolithographic methods offer excellent resolution of particle size and shape and have scalability comparable to that seen in the semiconductor industry, though the cost of equipment required for such facilities remains a hurdle to mass particle production. The PRINT method offers nanometer size scale resolution with control over particle size and shape and scalability in the form of a reel-to-reel production process. Of these methods, only PRINT and microfluidic methods have demonstrated their scalability, though hard template, photolithographic and particle stretching methods are all scalable in theory.

\section{Acknowledgments}

We thank the STC program of the NSF (CHE-9876674), the Carolina Center for Cancer Nanotechnology Excellence (NIH-U54-CA119343), ONR (N00014-07-1-0260), DARPA (07-4627), and Liquidia Technologies, Inc. for funding. Liquidia Technologies was recently launched by Joseph M. DeSimone and colleagues to commercialize breakthroughs from his laboratory, including micro and nanofluidics, soft lithography, and PRINT technology.

\section{REFERENCES}

1. Glotzer SC, Solomon MJ. Nat Mater. 2007; 6(8):557. [PubMed: 17667968]

2. Glotzer SC. Science. 2004; 306(5695):419. [PubMed: 15486279]

3. Lu Y, Yin Y, et al. Adv Mater. 2001; 13(6):415.

4. Beebe DJ, Moore JS, et al. Nature. 2000; 404(6778):588. [PubMed: 10766238]

5. Champion JA, Katare YK, et al. J Control Release. 2007; 121(1-2):3. [PubMed: 17544538]

6. Milliron DJ, Hughes SM, et al. Nature. 2004; 430(6996):190. [PubMed: 15241410]

7. Jana NR, Gearheart L, Murphy CJ. J Phys Chem B. 2001; 105:4065.

8. Manna L, Scher EC, Alivisatos AP. J Am Chem Soc. 2000; 122:12700.

9. Jin R, Cao Y, et al. Science. 2001; 294(5548):1901. [PubMed: 11729310]

10. Geng Y, Dalhaimer P, et al. Nat Nanotechnol. 2007; 2(4):249. [PubMed: 18654271]

11. Thurmond KB 2nd, Remsen EE, et al. Nucleic Acids Res. 1999; 27(14):2966. [PubMed: 10390540]

12. Hurst SJ, Payne EK, et al. Angew Chem Int Ed. 2006; 45(17):2672.

13. Hou S, Wang J, et al. J Am Chem Soc. 2005; 127(24):8586. [PubMed: 15954751]

14. Hou S, Wang J, et al. Nano Lett. 2005; 5(2):231. [PubMed: 15794602]

15. Dendukuri D, Doyle PS. Adv Mater. 2009; 21:1.

16. Nisisako T, Torii T. Lab Chip. 2008; 8(2):287. [PubMed: 18231668] 
17. Xu S, Nie Z, et al. Angew Chem Int Ed. 2005; 44(5):724.

18. Shepherd RF, Conrad JC, et al. Langmuir. 2006; 22(21):8618. [PubMed: 17014093]

19. De Geest BG, Urbanski JP, et al. Langmuir. 2005; 21(23):10275. [PubMed: 16262275]

20. Dendukuri D, Pregibon DC, et al. Nat Mater. 2006; 5(5):365. [PubMed: 16604080]

21. Shepherd RF, Panda P, et al. Adv Mater. 2008; 20(24):4734.

22. Pregibon DC, Toner M, et al. Science. 2007; 315(5817):1393. [PubMed: 17347435]

23. Hernandez CJ, Mason TG. J Phys Chem C. 2007; 111(12):4477.

24. Badaire S, Cottin-Bizonne C, et al. J Am Chem Soc. 2007; 129(1):40. [PubMed: 17199278]

25. 2009. www.liquidia.com

26. Canelas DA, Herlihy KP, et al. Wiley Interdis Rev: Nanomed Nanobiotech. 2009; 1(4):391.

27. Kelly JY, Desimone JM. J Am Chem Soc. 2008; 130(16):5438. [PubMed: 18376832]

28. Cai Z, Martin CR. J. Am. Chem. Soc. 1989; 111(11):4138.

29. Martin CR. Chem. Mater. 1996; 8(8):1739.

30. Brumlik CJ, Martin CR. J. Am. Chem. Soc. 1991; 113(8):3174.

31. Gasparac R, Kohli P, et al. Nano Lett. 2004; 4(3):513.

32. Hulteen JC, Martin CR. J. Mater. Chem. 1997; 7(7):1075.

33. Hou S, Harrell CC, et al. J. Am. Chem. Soc. 2004; 126(18):5674. [PubMed: 15125653]

34. Liang Z, Susha AS, et al. Adv. Mater. 2003; 15(21):1849.

35. Park S, Lim J-H, et al. Science. 2004; 303(5656):348. [PubMed: 14726585]

36. Martin BR, Dermody DJ, et al. Adv. Mater. 1999; 11(12):1021.

37. Banholzer MJ, Qin L, et al. Nat. Protoc. 2009; 4(6):838. [PubMed: 19444241]

38. Wei W, Li S, et al. Angew. Chem., Int. Ed. 2009; 48(23):4210.

39. Martin BR, Dermody DJ, et al. Adv Mater. 1999; 11(12):1021.

40. Banholzer MJ, Qin L, et al. Nat Protoc. 2009; 4(6):838. [PubMed: 19444241]

41. Christopher GF, Anna SL. J Phys D: Appl Phys. 2007; 40:R319.

42. Nisisako T, Torii T, et al. Lab Chip. 2002; 2(1):24. [PubMed: 15100856]

43. Anna SL, Bontoux N, Stone HA. Appl Phys Lett. 2003; 82:364.

44. Kenis PJ, Ismagilov RF, et al. Science. 1999; 285(5424):83. [PubMed: 10390366]

45. Cohen I, Li H, et al. Science. 2001; 292(5515):265. [PubMed: 11303097]

46. Zhihong, Nie; Shengqing, Xu; Minseok, Seo; Patrick, CLewis; Eugenia, Kumacheva. Journal of the American Chemical Society. 2005; 127(22):8058. [PubMed: 15926830]

47. Dendukuri D, Gu SS, et al. Lab Chip. 2007; 7(7):818. [PubMed: 17593999]

48. Decker C, Jenkins AD. Macromolecules. 1985; 18(6):1241.

49. Joanicot M, Ajdari A. Science. 2005; 309(5736):887. [PubMed: 16081724]

50. Nisisako T, Torii T, et al. Adv Mater. 2006; 18(9):1152.

51. Ho CC, Keller A, Odell JA, Ottewill RH. Colloid Polym Sci. 1993; 271:469.

52. Champion JA, Katare YK, et al. Proc Natl Acad Sci. 2007; 104(29):11901. [PubMed: 17620615]

53. Geissler M, Xia Y. Advanced Materials. 2004; 16(15):1249.

54. Jang J-H, Ullal CK, et al. Nano Letters. 2007; 7(3):647. [PubMed: 17295546]

55. Moon JH, Kim AJ, et al. Advanced Materials. 2007; 19(18):2508.

56. Badaire S, Cottin-Bizonne C, et al. Langmuir. 2008; 24(20):11451. [PubMed: 18788764]

57. Oudshoorn MHM, Penterman R, et al. Langmuir. 2007; 23(23):11819. [PubMed: 17927225]

58. Hernandez CJ, Kun Z, et al. Soft Mater. 2007; 5(1):13.

59. Gates BD, Xu Q, et al. Chemical Reviews. 2005; 105(4):1171. [PubMed: 15826012]

60. Jang J-H, Ullal CK, et al. Nano Lett. 2007; 7(3):647. [PubMed: 17295546]

61. Xia Y, Whitesides GM. Angew Chem Int Ed. 1998; 34(5):550.

62. Dumond JLHY. Adv Mater. 2008; 20(7):1291.

63. Glangchai LC, Caldorera-Moore M, et al. J Control Release. 2008; 125(3):263. [PubMed: 18053607] 
64. Guan J, Ferrell N, et al. Biomaterials. 2006; 27(21):4034. [PubMed: 16574217]

65. Moran IWC, Dalton F, Jhaveri SaravB, Carter KennethR. Soft Matter. 2008; 4:168.

66. Rolland JP, Maynor BW, et al. J Am Chem Soc. 2005; 127(28):10096. [PubMed: 16011375]

67. Zhang H, Nunes JK, et al. New J Phys. 2009; 11075018.

68. Gratton SE, Napier ME, et al. Pharm Res. 2008; 25(12):2845. [PubMed: 18592353]

69. Gratton SE, Pohlhaus PD, et al. J Control Release. 2007; 121(1-2):10. [PubMed: 17643544]

70. Gratton SE, Ropp PA, et al. Proc Natl Acad Sci. 2008; 105(33):11613. [PubMed: 18697944]

71. Herlihy KP, Nunes J, et al. Langmuir. 2008; 24(16):8421. [PubMed: 18646784]

72. Petros RA, Ropp PA, et al. J Am Chem Soc. 2008; 130(15):5008. [PubMed: 18355010]

73. Rolland JP, Van Dam RM, et al. J Am Chem Soc. 2004; 126(8):2322. [PubMed: 14982433]

74. Maynor BW, Larue I, et al. Small. 2007; 3(5):845. [PubMed: 17393549]

75. Xia Y, Rogers JA, et al. Chem Rev. 1999; 99(7):1823. [PubMed: 11849012]

76. Orgel R, Merkel TJ, et al. Unpublished work. 2008

77. Smithson R, McClure D, et al. Thin Solid Films. 1997; 307(1-2):110.

78. Glotzer SC, Solomon M. Nat Mater. 2007; 6(7):557. [PubMed: 17667968]

79. Sundararajan S, Lammert PE, et al. Nano Lett. 2008; 8(5):1271. [PubMed: 18416540] 


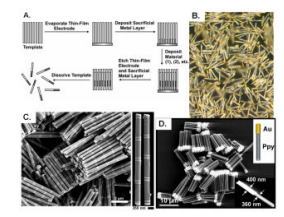

Figure 1.

(A) General scheme for nanorod synthesis via the deposition of materials into a nanoporous template, Copyright 2006 Wiley-VCH Verlag GmbH \& Co. KGaA. Reproduced with permission from [12]. (B) Bright-field optical micrographs of Au/Pt/Au rods. Reproduced with permission from [39], copyright 1999 Wiley-VCH Verlag GmbH \& Co. KGaA. (C) SEM images of Au-Ni multisegmented nanorods, Reprinted by permission from Macmillan Publishers Ltd: [Nature Protocols] [40], copyright (2009). (D) SEM image of Aupolypyrrole rods, From [35]. Reprinted with permission from AAAS. 


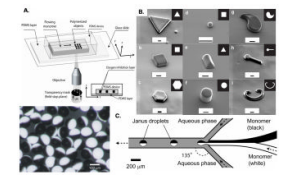

Figure 2.

(A) A schematic used for continuous-flow lithography and (B) microparticles of varying shapes fabricated with this method, adapted by permission from Macmillan Publishers Ltd: [Nature Materials] (20), copyright (2006). (C) Right: A schematic for the channel and flow configuration used for the formation of the bicolored janus particles shown on the left, reproduced with permission from [50], copyright 2006 Wiley-VCH Verlag GmbH \& Co. KGaA. 


\section{A}

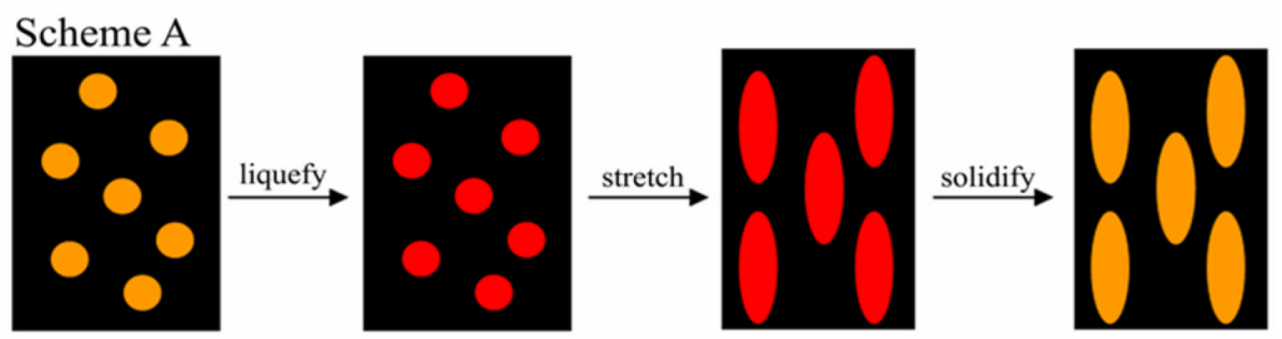

\section{Scheme B}
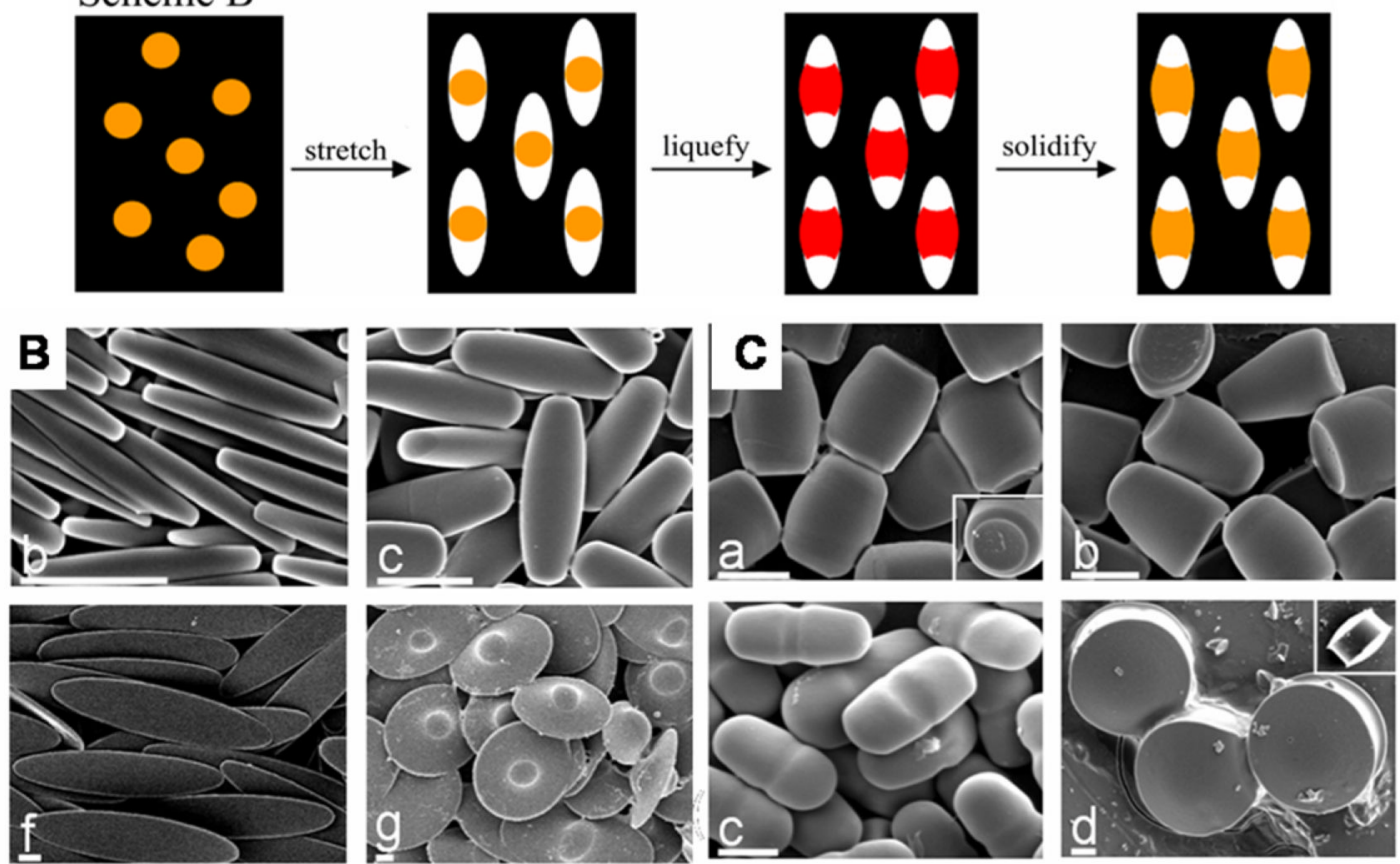

Figure 3.

(A) The two general schemes used in stretching spherical particles to different shapes. In Scheme A, particles are liquefied prior to stretching the film, while Scheme B has the film stretched prior to particle liquidification. (B) Polystyrene particles prepared via the method shown in scheme A. (C) Polystyrene particles prepared Scheme B. Figure adapted from reference [52], Copyright 2007 National Academy of Sciences, U.S.A. 

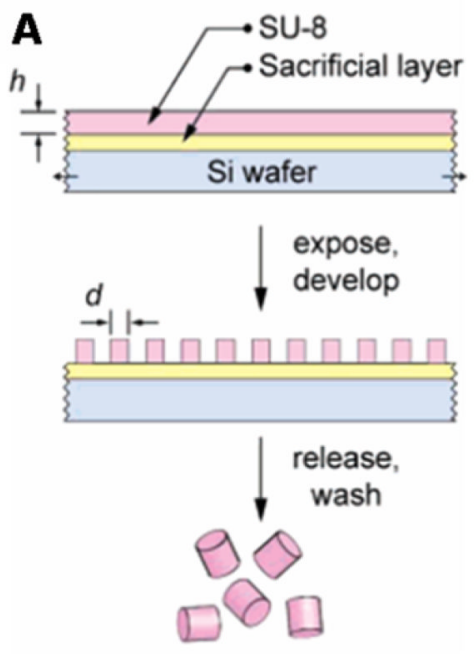

B
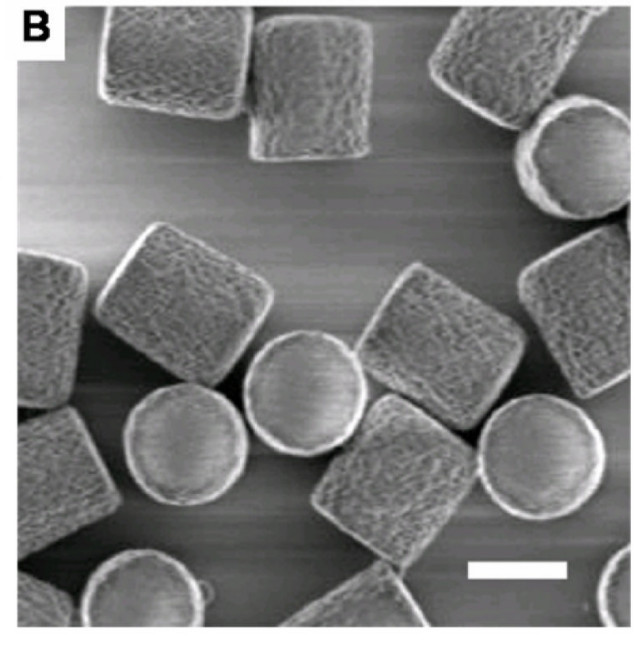
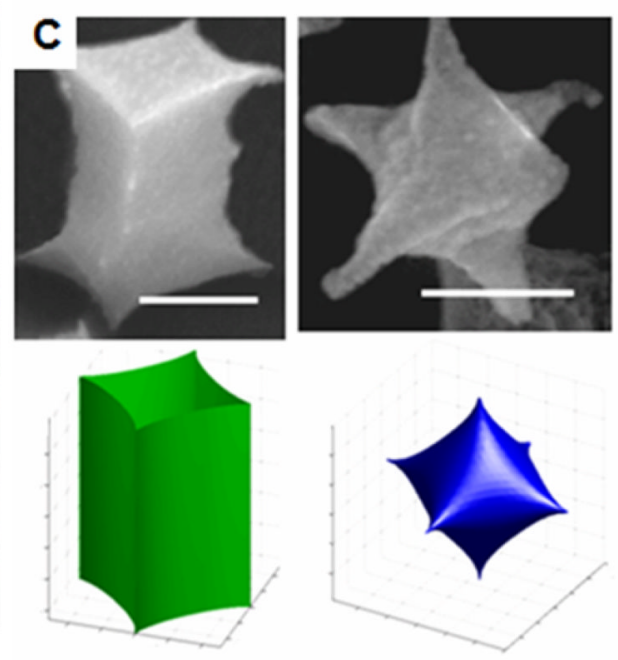

Figure 4.

(A) Simple scheme showing the photolithographic fabrication of colloidal particles, adapted with permission from [24], copyright 2007 American Chemical Society. (B) Cylindrical photoresist particles engineered with rough side walls (particle radius $=520 \mathrm{~nm}$, height $=$ $875 \mathrm{~nm}$ ), adapted with permission from [56], copyright 2008 American Chemical Society. (C) 4-valent and 6-valent nanoparticles fabricated using interference lithography (scale bars represent $300 \mathrm{~nm}$ ), adapted with permission from [60], copyright 2007 American Chemical Society. 
A

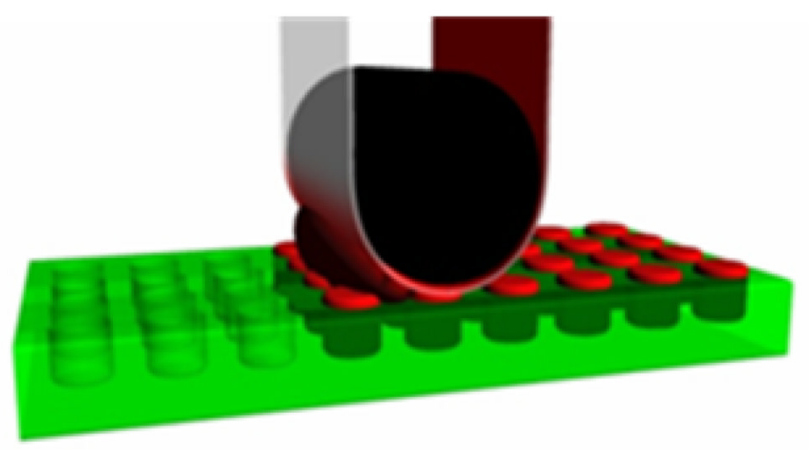

B

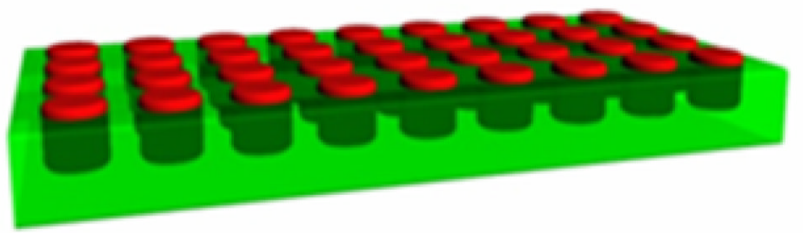

$C^{1}$

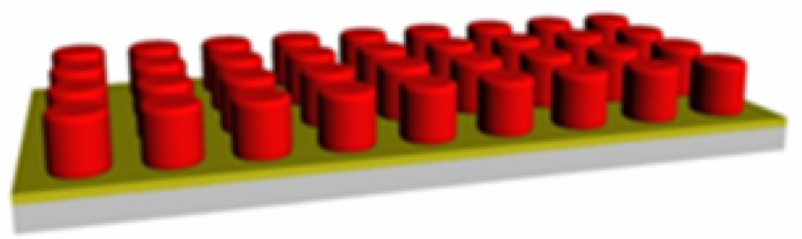

D)

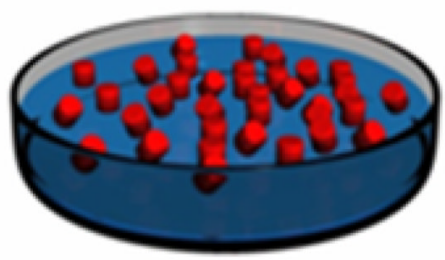

Figure 5.

A general schematic representation of the PRINT process. (A) A pre-particle solution (red) is distributed evenly in the elastomeric mold (green) by a roller (black) with a high surface energy polymer sheet, which wicks away excess solution. (B) The particle solution is solidified in the mold. (C) Particles are removed from the mold with a harvesting film (yellow). (D) Dissolution of the film yields free flowing particles in solution. 

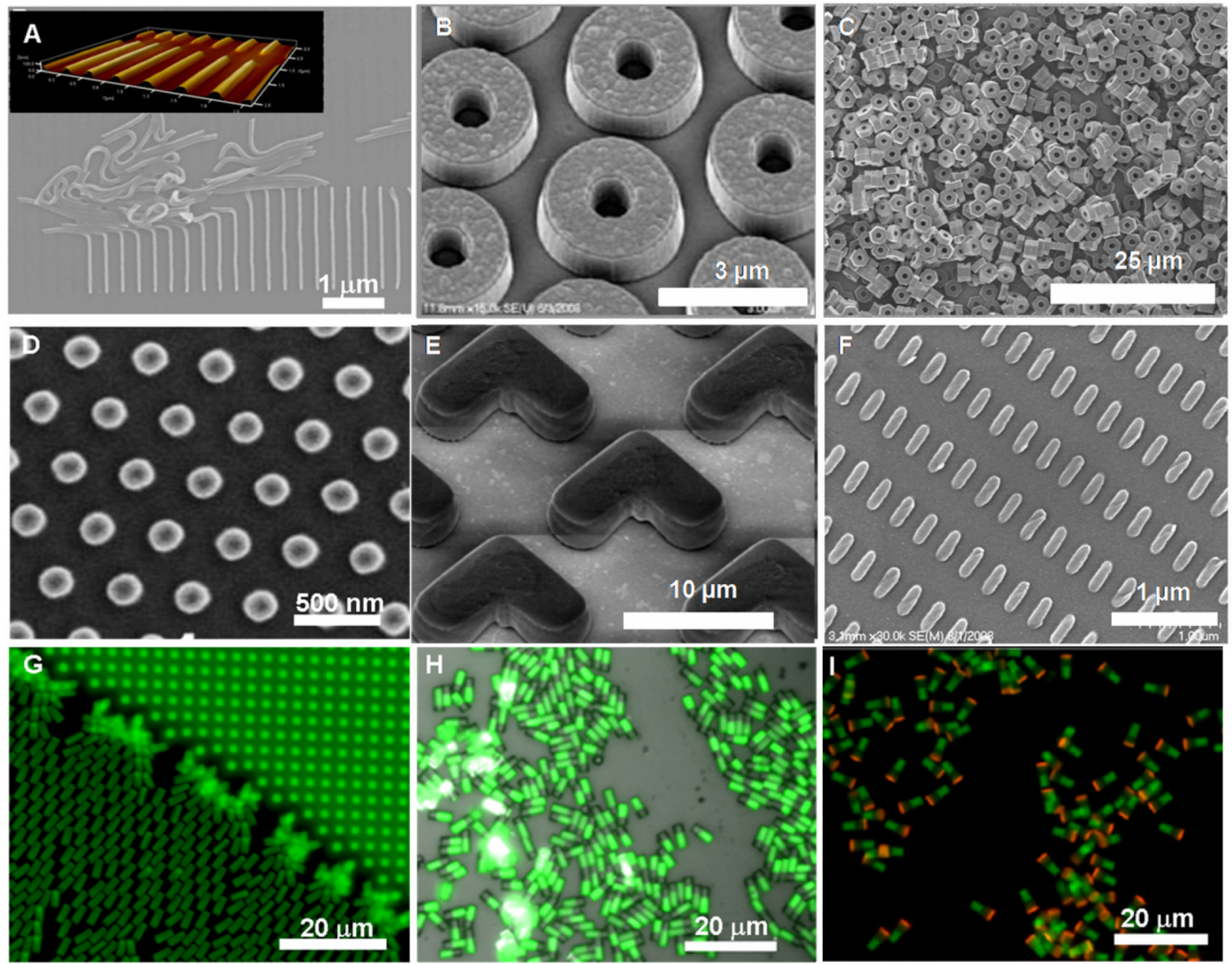

Figure 6.

Illustrative examples of size, shape, composition, cargo and surface chemistry control with the PRINT process (A) $80 \mathrm{~nm} \times 2000 \mathrm{~nm}$ worm-like cross-linked poly(ethylene glycol) (PEG) nanoparticles on a harvesting layer, inset showing AFM image of harvested layer, (B) $3 \mu \mathrm{m}$ toroids with roughened top surface made from cross-linked PEG (C) hexnut-shaped cross-linked PEG microparticles (D) siRNA-loaded $200 \mathrm{~nm}$ albumin nanoparticles harvested onto a medical adhesive, (E) boomerang-shaped cross-linked PEG microparticles on a harvesting layer, (F) $80 \times 360 \mathrm{~nm}$ cross-linked PEG particles on a polymeric harvesting layer, (G-I) $2 \times 2 \times 6 \mathrm{~mm}$ cross-linked PEG rectangular prisms showing increasing chemical anisotropy: $(\mathrm{G})$ one-phase, $(\mathrm{H})$ biphasic, and (I) triphasic. D is reprinted with permission from [27], copyright 2008 American Chemical Society. H, and I are reproduced with permission from reference [67], Copyright 2009 IOP publishing Ltd. 
Table 2

A comparison of swelling behavior, reported as the percent increase in weight of coupons of crosslinked elastomeric materials after soaking overnight. Traditional soft lithography mold material, poly (dimethylsiloxane) (PDMS), and PRINT mold material, perfluoropolyether (PFPE) were compared with regards to several solvents and polymerizable monomers76.

\begin{tabular}{lll}
\hline & $\begin{array}{l}\text { PDMS } \\
\text { (weight \% uptake) }\end{array}$ & $\begin{array}{l}\text { PFPE } \\
\text { (weight \% uptake) }\end{array}$ \\
\hline Water & $0.37 \pm 0.04$ & $0.39 \pm 0.16$ \\
Hexane & $109.82 \pm 1.41$ & $1.72 \pm 0.25$ \\
THF & $145.46 \pm 1.68$ & $6.95 \pm 0.08$ \\
DMSO & $2.36 \pm 0.50$ & $1.98 \pm 0.17$ \\
Isopropanol & $17.65 \pm 2.44$ & $2.38 \pm 0.06$ \\
Acetone & $20.15 \pm 1.05$ & $4.81 \pm 0.06$ \\
Poly (ethylene glycol) methyl ether & $1.04 \pm 0.45$ & $0.36 \pm 0.07$ \\
2-hydroxyethyl acrylate & $1.45 \pm 0.23$ & $1.03 \pm 0.22$ \\
N-Vinyl-2-pyrrolidone & $5.55 \pm 0.31$ & $2.34 \pm 0.27$ \\
PEG traicrylate & $1.40 \pm 0.06$ & $0.31 \pm 0.05$ \\
\hline
\end{tabular}

